\title{
Update PET in der Pädiatrischen Onkologie
}

\section{Update PET in pediatric oncology}

\author{
Autoren \\ Wolfgang Roll ${ }^{1}$, Uta Dirksen ${ }^{2,3}$, Matthias Weckesser ${ }^{1}$ \\ Institute \\ 1 Klinik für Nuklearmedizin, Universitätsklinikum Münster, \\ Münster \\ 2 Klinik für Kinderheilkunde III, Universitätsklinikum \\ Essen, Westdeutsches Tumorzentrum Essen, Essen \\ 3 Deutsches Konsortium für Translationale \\ Krebsforschung (DKTK), Standort Essen und Cancer \\ Centre Cologne Essen (CCCE), Essen
}

Schlüsselwörter

PET/CT, PET/MRT, Pädiatrische Onkologie

Key words

$\mathrm{PET} / \mathrm{CT}, \mathrm{PET} / \mathrm{MRI}$, pediatric oncology

\author{
Bibliografie \\ DOI https://doi.org/10.1055/a-0621-3182 | \\ Der Nuklearmediziner 2018; 41: 211-221 \\ (c) Georg Thieme Verlag KG Stuttgart · New York \\ ISSN 0723-7065
}

\author{
Korrespondenzadresse \\ Dr. med. Wolfgang Roll, Klinik für Nuklearmedizin, \\ Universitätsklinikum Münster, Albert-Schweitzer- \\ Campus 1, Gebäude A1, 48149 Münster, Germany \\ Tel.: +49/2 51/8347362 \\ Fax: $+49 / 251 / 8347363$ \\ Wolfgang.Roll@ukmuenster.de
}

\section{ZUSAMMENFASSUNG}

Die PET/CT wird bei vielen kindlichen Tumorentitäten eingesetzt und hat beim Hodgkin-Lymphom bereits Eingang in nationale und internationale Leitlinien gefunden. Die PET ermöglicht eine semiquantitative Messung der metabolischen Tumoraktivität und damit eine frühe Beurteilung des Therapieansprechens sowie der Vitalität von Tumorrestgewebe oder eines Rezidivs. Für verschiedene Fragestellungen stehen unterschiedliche metabolische und molekulare Marker zur Verfügung.

In dieser Übersichtsarbeit werden Indikationen für PET im Kindesalter sowie die besonderen Anforderungen in der Durchführung und Befundung dargelegt. Abschließend wird die Bedeutung der neuen PET/MRT für die Diagnostik kindlicher Tumore diskutiert.

\section{ABSTRACT}

$\mathrm{PET} / \mathrm{CT}$ is used in many pediatric malignancies and is part of international guidelines for Hodgkin-lymphoma imaging. PET is useful for the evaluation of metabolic tumor activity. Thus it is helpful for the early determination of response to therapy and to delineate vital tumor tissue in residual or recurrent tumor. This review outlines different indications for PET in childhood using targeted molecular probes. Age related patient preparation and patterns of tracer distribution need special consideration during image analysis. The incremental value of PET/MRI for the imaging of pediatric malignancies is discussed.

\section{Einleitung}

Die Positronen-Emissions-Tomografie (PET) kombiniert mit morphologischer Computer(CT)- oder magnetresonanztomografischer (MRT)-Bildgebung gewinnt für das Staging und Management pädiatrischer Tumorerkrankungen an Bedeutung. In der klinischen Routine werden insbesondere bei Hodgkin-/NonHodgkin-Lymphomen, Knochen- und Weichteilsarkomen, KopfHals-Tumoren inklusive Schilddrüsenkarzinomen und ZNS-Tumoren die PET eingesetzt. Neben der auch im Kindesalter am häufigsten eingesetzten ${ }^{18}$ Fluor-2-fluoro-2-deoxy-d-glucose (FDG), werden, je nach Indikation, Tracer zur Darstellung anderer metabolischer und molekularer Vorgänge, wie bspw. des Aminosäuretransports ( ${ }^{18} \mathrm{~F}$-Fluorethyltyrosin, FET), genutzt.
Bei der Durchführung der PET-Untersuchung im Kindesalter gilt es einige Besonderheiten zu beachten. Diese betreffen nicht nur das Management der Kinder und ihrer Eltern, sondern auch die notwendigen Anpassungen der Technik an den kleinen Patienten. Bei der Befundung der FDG-PET sind Unterschiede in der Verteilung der Stoffwechselaktivität gegenüber Erwachsenen zu beachten.

Die seit einigen Jahren verfügbare PET/MRT erlaubt die integrierte Bildakquisition zweier in der pädiatrischen Bildgebung etablierter Verfahren, der Ganzkörper(GK)-PET und der MRT bei gleichzeitig geringerer Strahlenexposition durch die fehlende CT-Komponente. 


\section{Indikationen}

Die Liste der Indikationen für PET-Untersuchungen in der pädiatrischen Onkologie erweitert sich fortlaufend. Die S3-Leitlinie zur Ganzkörper-FDG-PET-Bildgebung bei Kindern mit onkologischen Erkrankungen wird im Moment aktualisiert. Für den Einsatz anderer PET-Tracer bei Kindern liegen keine eigenen Leitlinien vor.

\section{Hodgkin- und Non-Hodgkin-Lymphome}

Tumore des lymphatischen Systems sind die dritthäufigste Gruppe von Tumoren im Kindes- und Jugendalter und lassen sich in Hodgkin- und Non-Hodgkin-Lymphome einteilen. Die Non-Hodgkin-Lymphome wiederum lassen sich in T- und BZell-Lymphome unterteilen [1, 2].

\section{Hodgkin-Lymphome}

Das Auftreten des Hodgkin-Lymphoms im Kindes und Jugendalter ist altersabhängig und betrifft in den westlichen Industrieländern vor allem die Gruppe der 15- bis 19-Jährigen. Bei Erstdiagnose liegt in den meisten Fällen eine schmerzlose Lymphadenopathie, besonders häufig supraklavikulär, zervikal oder mediastinal vor. Der Befall ist primär nodal und die Ausbreitung erfolgt über weitere Lymphknotenstationen. Milz, Leber, Lunge und Knochenmark sind in ungefähr 15-20\% sekundär befallen. Die Stadieneinteilung erfolgt nach Ann-Arbor, welche sich wesentlich auf die Lokalisation der befallenen Lymphknotenstationen mit dem Zwerchfell als Barriere stützt. Die Prognose ist bei entsprechender Behandlung mit einer 5-Jahres-Überlebensrate von über $90 \%$ exzellent [2].

Eine der am besten erforschten Indikationen für die PET-Bildgebung ist die Darstellung des Hodgkin-Lymphoms ( $\mathbf{A b b}$. 2). Neben dem initialen Staging und der Beurteilung des Therapieansprechens nach Therapie wird auch die Bestrahlungsplanung bei Hodgkin-Lymphomen mittels FDG-PET/CT durchgeführt. Die Bewertung der metabolischen Response schon früh nach Therapiebeginn durch die Darstellung der verbleibenden Stoffwechselaktivität mittels FDG bestimmt dabei maßgeblich das klinische Procedere und ist auch prädiktiv für den weiteren Verlauf der Erkrankung [2 -5].

\section{Non-Hodgkin-Lymphome}

Im Kindes- und Jugendalter treten im Unterschied zum Erwachsenen vor allem aggressive Lymphome, wie das lymphoblastische-, diffus großzellige B-Zell-, Burkitt-, oder großzellig anaplastische Lymphom auf. Niedrigmaligne Lymphome kommen nur selten vor. Die Non-Hodgkin-Lymphome zeigen im Kindesalter, im Gegensatz zum Erwachsenenalter, häufiger einen extranodalen Befall (Mediastinum, Kopf-Hals-Region, Abdomen, Knochenmark, ZNS), bspw. eine abdominelle Raumforderung beim Burkitt-Lymphom. Bei Non-Hodgkin-Lymphomen ist die St.Jude-/Murphy-Stadieneinteilung am weitesten verbreitet, die sich am extranodalen Befall orientiert. Die Prognose hat sich genau wie beim Hodgkin-Lymphom in den letzten Jahren deutlich verbessert mit 5-Jahres-Überlebensraten zwischen 80 und $90 \%[1]$.
Insbesondere bei Kindern häufig vorkommende aggressive Lymphome, zeigen sich auf hohem Niveau FDG-positiv [6,7]. Ziel der FDG-PET sind analog zum Hodgkin-Lymphom das initiale Staging mit höherer Sensitivität gegenüber morphologischen Bildgebungsmethoden und die Bewertung der frühen metabolischen Response. Zur Beurteilung des Therapieansprechens wird, wie auch bei Hodgkin-Lymphomen, der Deauville 5point score eingegliedert in die Lugano-Klassifikation genutzt, der sich an der FDG-Avidität der Tumormanifestationen im Vergleich zum mediastinalen Blutpool und der Aktivität in der Leber orientiert [5, 8]. Nicht FDG-avide Non-Hodgkin-Lymphome werden primär mittels KM-gestütztem CT und mit der MRT untersucht $[5,7]$.

\section{Sarkome}

Sarkome sind maligne Tumore mesenchymalen Ursprungs. Knochen- und Weichteilsarkome haben im Kindes- und Jugendalter einen Anteil von $12 \%$ an allen malignen Erkrankungen. Die häufigsten primären Knochentumore sind das Osteosarkom und das Ewing-Sarkom. Diese kommen insbesondere im Jugend- und jungen Erwachsenenalter vor. Die häufigsten Weichteilsarkome des Kindes- und Jugendalters sind alveoläre/embryonale Rhabdomyosarkome. Die Heilungsraten pädiatrischer Knochen- und Weichteilsarkome liegen im lokalisierten Stadium über $70 \%$, wohingegen die Prognose im metastasierten Stadium weiterhin schlecht ist [9-12].

\section{Osteosarkom}

Osteosarkome sind meist osteoidproduzierende primäre Knochentumore, die häufig die Metaphyse der langen Röhrenknochen, besonders den distalen Femur, die proximale Tibia und den proximalen Humerus, betreffen. Die Altersverteilung der Inzidenz zeigt 2 Häufigkeitsgipfel, einen im jugendlichen Alter und einen zweiten bei älteren Erwachsenen über 65 Jahren. Häufige Lokalisationen von Metastasen, die in 10-20\% der Fälle vorkommen, sind, neben den lokoregionären Lymphknoten, insbesondere pulmonale Metastasen und Knochenmetastasen [10].

Bei Osteosarkomen zeigen sich prognostisch relevante Metastasen, insbesondere der Lunge aber auch des Knochens, teilweise ohne signifikante FDG-Aufnahme. Eine stoffwechselaktive Darstellung des Primärtumors ist bei dieser Erkrankung nicht immer mit einer ähnlich stoffwechselaktiven Metastasierung assoziiert. Verfahren der Wahl zum Ganzkörperstaging sind daher die Skelettszintigrafie und die MRT, kombiniert mit morphologischer Bildgebung der Lunge (CT) $[13,14]$. Häufig zitierte Studien, die die Bedeutung der FDG-PET für die Detektion von Osteosarkommetastasen unterstreichen, ziehen Rückschlüsse aus Mischkollektiven, die nicht hinreichend durch Daten belegt werden können $[15,16]$. In der angeführten Studie sind nur wenige Osteosarkompatienten eingeschlossen worden, mit konsekutiv geringer Zahl an Lymphknotenmetastasen $(n=1)$ und Patienten mit Knochenmetastasen $(n=4)[15,16]$.

Die morphologische Darstellung des Primärtumors mit der Frage nach Infiltration benachbarter Strukturen erfolgt analog zu anderen Sarkomtypen durch die MRT. Zusätzlich kann mittels FDG-PET die Vitalität des Primärtumors quantitativ be- 

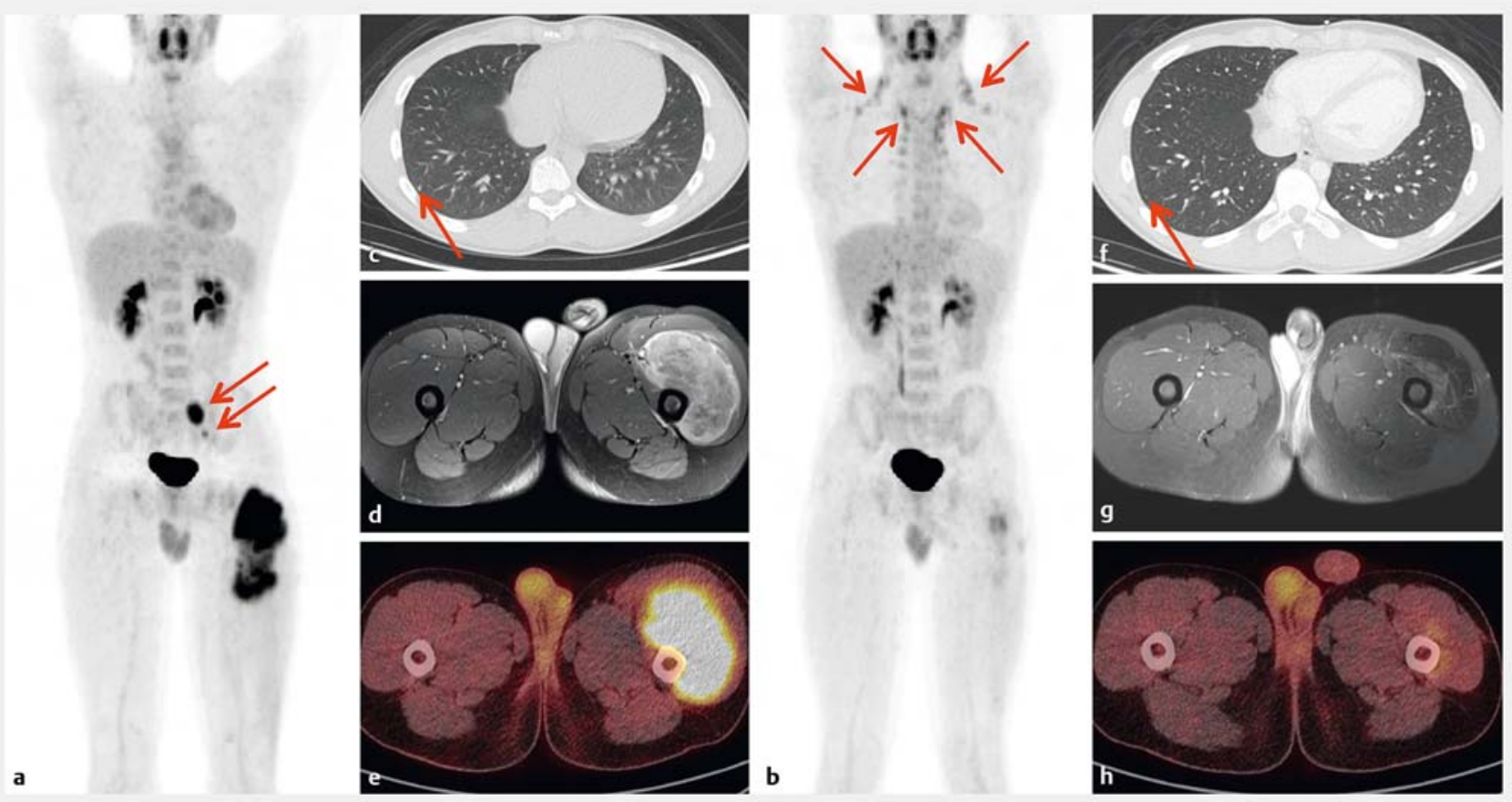

- Abb. 1 PET/CT Staging eines 14-jährigen Patienten mit einem Ewing-Sarkom im proximalen linken Femur. Der Primärtumor zeigt sich in der Ganzkörperaufnahme (a) und der Fusion (e) mit deutlich vermehrter FDG-Anreicherung und teilweise nekrotischen, nicht stoffwechselaktiven Arealen. Im korrespondierenden MRT zeigt sich der Tumor in der T1-gewichteten Sequenz deutlich KM-aufnehmend (d). Im Ganzkörperstaging stellen sich zusätzlich iliakale Lymphknoten (Pfeile) mit vermehrter Nuklidanreicherung im Sinne von Lymphknotenmetastasen dar. In der Niedrigdosis-CT zeigt sich ein kleiner subpleuraler Rundherd mit $5 \mathrm{~mm}$ im rechten Unterlappen (c), im Rahmen der Grunderkrankung als metastasensuspekt gewertet.

Im Staging nach 2 von 6 Zyklen Chemotherapie kann ein sehr gutes Therapieansprechen mit nur noch geringer Nuklidbelegung des Primärtumors $(\mathbf{b}, \mathbf{h})$ und einer deutlichen Größenabnahme in der MRT $(\mathbf{g})$ gezeigt werden. Die iliakalen Lymphknotenmetastasen, die in der prätherapeutischen Ganzkörperaufnahme (a) noch deutlich auszumachen waren, kommen unter Chemotherapie in den Ganzkörperaufnahmen (e) nicht mehr zur Darstellung. Der als metastasensuspekt vorbeschriebene Rundherd pulmonal lässt sich nur noch angedeutet abgrenzen (f).

In der Ganzkörperaufnahme unter Chemotherapie (b) zeigt sich trotz Prämedikation mit einem Betablocker eine leicht vermehrte Nuklidanreicherung im braunen Fettgewebe (Pfeile).

stimmt und eine Prognoseabschätzung aufgrund der Veränderungen der metabolischen Bildgebungsparameter gegeben werden [17-19].

Dieser quantitative PET-Ansatz mit Bestimmung und Evaluation der Total Lesion Glycolysis (TLG) und des metabolischen Volumens (MV) aller Tumorläsionen in Bezug auf Response oder/und Outcome zeigt vielversprechende Ergebnisse für alle Sarkome, die in großen prospektiven Studien bestätigt werden müssen [17, $20-22]$.

\section{Ewing-Sarkome}

Ewing-Sarkome treten meist im Knochen auf, können jedoch auch im Weichteilgewebe entstehen, letzteres vor allem bei älteren Patienten. Häufigste Lokalisation sind das Becken sowie die untere Extremität, insbesondere der Femur, gefolgt vom Rippenskelett. Bei einem Viertel der Patienten liegen zum Zeitpunkt der Erstdiagnose bereits Metastasen, jeweils zur Hälfte in Lunge oder Knochen, vor. Die Therapie des Ewing-Sarkoms erfolgt im Rahmen von Studien mittels neoadjuvanter Chemotherapie, gefolgt von einer Resektion und histologischen Aufarbeitung [12,23]. Zur Therapieplanung ist eine genaue morpholo- gische Darstellung des Primärtumors erforderlich. Therapierelevante Fragestellungen sind die Tumorausbreitung, die Infiltration benachbarter Strukturen durch die Weichteilkomponente, eine mögliche Gelenkbeteiligung und der Nachweis diskontinuierlicher Zweitläsionen im gleichen Kompartiment sog. „Skip-Lesions“. Darüber hinaus ist ein sorgfältiges und vollständiges initiales Ganzkörperstaging notwendig, da das Therapiekonzept häufig eine Lokaltherapie auch von Metastasen beinhaltet [24]. Für die Operationsplanung und die primäre lokale Diagnostik ist, je nach Lokalisation, ein MRT oder CT (Thorax) unerlässlich. Für das Ganzkörperstaging stellt das FDG-PET/CT ein sehr gutes Verfahren zur Darstellung von Knochenmetastasen (PET) und eines Lungenbefalls (CT) dar ( $\triangleright$ Abb. 1) $[14,16$, 25, 26].

Die Bedeutung quantitativer PET-Parameter für eine Prognoseabschätzung ist zum jetzigen Zeitpunkt nicht abschließend geklärt. In mehreren retrospektiven Untersuchungen war eine Abschätzung der Prognose hinsichtlich progressionsfreiem bzw. eventfreiem Überleben ausgehend vom initialen maximalen SUV möglich [27, 28]. Eine der ersten Studien von Hawkins konnte diese Korrelation jedoch nur zwischen dem SUV max $_{\max }$ 



- Abb. 2 Initiales PET/MRT-Staging einer 16-jährigen Patientin mit Hodgkin Lymphom (Stadium II, Ann-Arbor-Klassifikation). In den Ganzkörperaufnahmen (a) und der Fusion (b) zeigen sich die zervikalen, mediastinalen und bihilären Lymphknoten mit einem maximalen Uptake von SUV: 12.1 bei einem Leberreferenzwert von SUV:1.8. In der koronaren T2-gewichteten (c) sowie den transversalen T1-gewichteten Sequenzen mit KM (d, e) ist die zervikale und mediastinale Lymphadenopathie markiert (Pfeile).

des Stagings nach neoadjuvanter Chemotherapie und dem progressionsfreien Überleben sowie dem histologischen Ansprechen zeigen [17]. Raciborska et al. bestätigen den Zusammenhang zwischen posttherapeutischen SUV max $_{\text {max }}$ und Outcome und konnte einen Zusammenhang zwischen initialem SUV max $_{\text {und }}$ histologischem Ansprechen zeigen. Einen Zusammenhang zwischen metabolischem Ansprechen, also der Veränderung der quantitativen PET-Parameter, unter Chemotherapie und histologischem Ansprechen kann von einigen Arbeitsgruppen bestätigt werden $[17,29]$, wobei Palmeri et al. im Gegensatz zu Hawkins et al. sogar einen Einfluss auf das eventfreie Überleben zeigen konnten $[17,28]$. Einschränkend gilt, dass die vorliegenden Studien in den meisten Fällen retrospektiv ausgewertet wurden und teilweise nur sehr geringe Patientenzahlen, knapp über 10 Ewing-Sarkom-Patienten, aufweisen. Zusammenfassend gilt, dass die zum Teil kontroversen Ergebnisse der dargelegten Studien durch große prospektive Studien validiert werden müssen. Eine prospektive Studie zur Auswertung dieser Fragen wird derzeit im Rahmen der EWING 2008 Studie durchgeführt [30].

Nach erfolgreicher Behandlung des Ewing-Sarkoms sind regelmäßige Nachsorgeuntersuchungen notwendig. Die bildgebende Darstellung des Rezidivs im Rahmen des Nachsorgeprotokolls zeigt, insbesondere bei Rezidiven der Lunge, einen signifikanten Vorteil im Gesamtüberleben gegenüber der Rezidiv- detektion bei symptomatischen Patienten außerhalb des geplanten Nachsorgeprotokolls [31]. Vor dem Auftreten von Symptomen können die meisten Rezidive durch FDG-PET/CT im Vergleich zu konventionellen Bildgebungsmethoden detektiert werden [32]. Die Detektion von Rezidiven gelingt mittels PET/CT dabei mit sehr hoher Genauigkeit [33,34]. Die im PET/ CT integrierte Low-Dose-CT in Inspiration zeigte sich suffizient zur Erkennung kleiner pulmonaler Herde [32].

\section{Rhabdomyosarkom}

Rhabdomyosarkome des Kindes- und Jugendalters lassen sich histologisch in einen alveolären und einen embryonalen Subtyp einteilen. Andere (z.B. pleomorphe) Subtypen liegen selten vor. Die Lokalisation und das Alter bei Erstdiagnose erlauben Rückschlüsse auf die wahrscheinlichste Histologie und Prognose des Primärtumors. Rhabdomyosarkome können im gesamten Körper vorkommen. Bei jüngeren Kindern sind Kopf-HalsTumore des embryonalen Typs häufig, wohingegen Extremitätentumore häufiger Jugendliche betreffen und dem alveolären Typ zugehörig sind. Tumore der Harn- und Geschlechtsorgane sind in den meisten Fällen vom embryonalen Typ, welcher sich weniger aggressiv und mit geringerer Tendenz zum Rezidiv als der alveoläre Subtyp zeigt. Metastasen der Rhabdomyosarkome 



- Abb. 3 FET-PET/MRT eines 3-jährigen Kindes mit einem desmoplastischen Medulloblastom im Bereich der hinteren Schädelgrube. Die infratentorielle Raumforderung (d) zeigt eine deutlich vermehrte Nuklidanreicherung bei gleichzeitig geringem Hintergrundsignal im umgebenden Hirngewebe im PET (a), der MIP (b) sowie der Fusion aus PET und MRT (c). In der MIP zeigen sich keine weiteren fokalen Nuklidanreicherungen im Hirnparenchym.

zeigen sich vorwiegend in den lokoregionären Lymphknoten, pulmonal und im Skelettsystem [9, 12].

Für das Primärstaging pädiatrischer Rhabdomyosarkome liegen im Gegensatz zum Therapiemonitoring mittels FDG-PET bereits einige Studien und eine Metaanalyse vor. Norman et al. kommen in ihren 8 Studien und insgesamt 272 Rhabdomyosarkom-Patienten unter 24 Jahre umfassenden Metaanalyse zu dem Schluss, dass Sensitivität und Spezifität der Detektion von Lymphknoten und Fernmetastasen mittels FDG-PET/CT deutlich höher war, als mit konventionellen Bildgebungsmethoden [35]. Eine Unterscheidung zwischen Fernmetastasen nach Lokalisation war bei geringer Fallzahl nicht möglich. Angenommen werden kann jedoch, dass die PET für die Erkennung von Knochen-, Weichteilmetastasen und einer Knochenmarksinfiltration konventionellen Bildgebungsmethoden in den Punkten Sensitivität und Spezifität überlegen ist [34-36]. Bez. der Detektion von Lungenmetastasen ist die CT- der PET-Komponente überlegen. Aufgrund der geringeren Anzahl an postentzündlichen Rundherden bei Kindern im Vergleich zu Erwachsenen ist auch die Spezifität der CT für Lungenmetastasen hoch. Für das initiale Staging zeigt die Kombination beider Verfahren im PET/ CT somit eine hohe diagnostische Sicherheit und sollte das Verfahren der Wahl darstellen [34].

Wie bereits für andere Sarkome beschrieben, hat die Intensität des Stoffwechsels des Primärtumors zum Zeitpunkt der Erstdiagnose eine prognostische Bedeutung [37].

Bez. einer Vorhersage der Überlebensrate anhand von frühem Therapieansprechen in der PET kann auf Basis der aktuellen Datenlage keine eindeutige Aussage getroffen werden. Eine Studie von Casey et al. zeigt bspw. eine Korrelation zwischen metabolischem Ansprechen im PET und progressionsfreiem Überleben, wohingegen erste prospektive Studiendaten der Gruppe um Harrison et al. keine Korrelation zu eventfreiem Überleben finden konnten [34, 38, 39].

\section{Neuroblastome}

Neuroblastome gehören zur Gruppe der embryonalen Tumore und sind die häufigsten extrakraniellen Tumore des Kindesalters. Betroffen sind am häufigsten das Nebennierenmark und die abdominellen, paraaortalen oder intrathorakalen Paraganglien. Häufigste Erstmanifestation ist ein retroperitonealer, abdomineller Tumor. Die Metastasierung erfolgt bereits nach kurzer Zeit in Skelett, Leber oder generalisiert und liegt in 70\% der Fälle zum Zeitpunkt der Diagnosestellung vor. Die Prognose ist altersabhängig und mit $90 \%$ 5-Jahres-Überlebensrate bei Kindern unter einem Jahr exzellent, wobei Patienten zwischen 1 und 14 eine vergleichsweise geringe 5-Jahres-Überlebensrate von $68 \%$ aufweisen. Bei Säuglingen unter einem Jahr ist eine spontane Rückbildung möglich [40].

Das Standardverfahren zum Primärstaging und zur Beurteilung des Therapieansprechens von Neuroblastomen ist die ${ }^{123}$ Metaiodobenzylguanidin(MIBG)-Szintigrafie. Bei MIBG-negativen Tumoren oder komplementär in fortgeschrittenen Stadien kann eine FDG-PET-Untersuchung durchgeführt werden [14]. Erste Studien weisen zudem daraufhin, dass eine initial hohe FDG-Anreicherung mit einer schlechteren Prognose korreliert [41]. Die Bedeutung von Tracern für die Darstellung der Somatostatinrezeptor-Expression (DOTATATE) oder für das sympathische Nervensystem (HED: Hydroxyephedrin, DOPA: Dihydroxyphenylalanin) ist für die Bildgebung der Neuroblastome im Kindesalter nicht abschließend geklärt $[42,43]$.

\section{Hirntumore}

Die Häufigkeit histologischer Subtypen, die Therapie und die Prognose kindlicher Hirntumore unterscheiden sich wesentlich von der Situation bei Erwachsenen. ZNS-Tumore sind die häufigste Entität solider Tumore im Kindesalter und haben die höchste Mortalität aller malignen Erkrankungen in dieser Altersgruppe. Im Kindes- und Jugendalter liegt der Anteil maligner Hirntumore an den Hirntumoren um 2 Drittel, wohingegen nur ungefähr ein Drittel der Hirntumore im Erwachsenenalter 
maligne sind. Der häufigste Hirntumor im Kindes- und Jugendalter ist das pilozytische Astrozytom, ein benignes Gliom, gefolgt von malignen Gliomen, embryonalen Tumoren, insbesondere dem Medulloblastom, sowie Tumore der Hirnanhangsdrüse. Im Erwachsenenalter stellen Metastasen die größte Zahl der malignen Raumforderungen im Gehirn dar, wohingegen bei Kindern und Jugendlichen hirneigene Tumore dominieren. Der häufigste maligne Tumor des Kindesalters ist das Medulloblastom, welcher ausschließlich das Kleinhirn betrifft und beim Erwachsenen ab der vierten Lebensdekade aufgrund des embryonalen Ursprungs nahezu nicht mehr vorkommt. Die häufigste Lokalisation aller Tumore im Kindesalter $(0-14)$ ist die hintere Schädelgrube, wohingegen bei Jugendlichen zwischen 15 und 19 Jahren Tumore der Hirnanhangsdrüse gefolgt vom Cerebellum am häufigsten vorkommen. Im Erwachsenenalter kommen Tumore im Bereich der hinteren Schädelgrube nur äußerst selten vor, sondern betreffen meist die Meningen, die Hirnanhangsdrüse oder den Neokortex [44].

Ziel der Diagnostik von Hirntumoren ist es, die genaue anatomische Lokalisation und den Bezug zu funktionell wichtigen Hirnarealen darzustellen sowie eine Differenzialdiagnostik zu nicht neoplastischen Hirnraumforderungen zu ermöglichen [45]. Initial wird im Regelfall eine MRT-Untersuchung mit Kontrastmittel (KM) durchgeführt. Einschränkungen sind das teilweise unterschiedliche, teilweise identische KM-AufnahmeVerhalten der verschiedenen benignen und malignen Hirntumore und die eingeschränkte Differenzierungsmöglichkeit vitalen Tumors von Gliose oder Ödem. Das pilozytische Astrozytom bspw., der häufigste Hirntumor des Kindesalters, benigne und mit guter Langzeitprognose, zeigt genau wie maligne Gliome eine Kontrastmittelanreicherung. In Verlaufskontrollen zum Therapieansprechen oder zur Rezidivdiagnostik sind therapiebedingte Schrankenstörungen in der MRT nicht sicher von vitalem Tumorrestgewebe zu unterscheiden. Insbesondere die Darstellung dieses vitalen Tumorgewebes mit der PET ist von auBerordentlicher Bedeutung für die Planung des weiteren Therapieverlaufs mit stereotaktischer Biopsie/Resektion [46-48] oder Bestrahlung (ESRT) [49, 50].

Der FDG-Uptake in die Hirntumore korreliert bei Erwachsenen zwar mit dem Malignitätsgrad und besitzt eine prognostische Bedeutung $[46,51]$. Im Kindesalter trifft diese Regel allerdings nur bedingt zu, da z. B. die o.g. pilozytischen Astrozytome auch einen gesteigerten Glukosestoffwechsel aufweisen können. Auch ist der Tumorkontrast aufgrund des ausgeprägten Glukosestoffwechsels des angrenzenden gesunden Hirngewebes, nicht nur bei niedrig malignen Gliomen unzureichend. Daher werden heute Tracer zur Darstellung des Aminosäurestoffwechsels (FET, DOPA) eingesetzt ( Aminosäure-PET kann in vielen Fällen zwischen therapiebedingten Gewebsveränderungen und Tumorrezidiven/Resttumorgewebe unterscheiden und zur Beurteilung des Ansprechens auf die Therapie eingesetzt werden [55-57].

Die publizierten Daten zur Wertigkeit der PET mit FDG oder Tracern zur Darstellung des Aminosäure-Stoffwechsels (FET) bei Kindern stützen sich bis auf wenige Ausnahmen [48,52, 53] maßgeblich auf Ergebnisse von Untersuchungen am Erwachsenen. Die Ergebnisse einer prospektiven, multizentri- schen Studie (FET-PET 2010) zur Evaluation der FET-PET gegenüber der konventionellen MRT bei Kindern stehen noch aus [58].

\section{Seltene Tumore}

Weitere seltene Indikation für PET im Kindes- und Jugendalter sind bspw. die frühkindliche Histiozytose, entdifferenzierte Schilddrüsenkarzinome oder die Neurofibromatose Typ I mit der Frage nach einer malignen Transformation. Aufgrund der Seltenheit der Erkrankungen liegt nur wenig Evidenz für die PET bei Kindern vor. Die vorhandene Datenlage stützt sich hauptsächlich auf Studien bei Erwachsenen oder gemischten Kollektiven.

\section{Histiozytose}

Histiozytäre Veränderungen sind durch klonale Proliferation verschiedener Zellen des mononukleären Phagozytensystems gekennzeichnet. Die bekannteste Form der Langerhans-Histiozytose zeigt sich durch Akkumulation aktivierter dendritischer Zellen und Makrophagen in einem oder mehreren Organen oder disseminiert. Betroffen sind hauptsächlich Kinder unter 15 Jahren, von denen wiederum Kinder unter 2 Jahren häufig zur Gruppe der Hochrisiko-Patienten gehören. Die Einteilung in Hoch- und Niedrigrisiko-Patienten erfolgt nach befallenen Organen, wobei die am häufigsten befallenen Organe, Haut und Knochen, zu den Niedrigrisiko-Organen gerechnet werden [59]. Die Bedeutung der PET bei malignen histiozytären Veränderungen des Kindesalters liegt im primären Staging sowie der Rezidivdiagnostik mittels FDG [60-62]. Zudem kann das Therapieansprechen in der PET mit größerer Genauigkeit im Vergleich zu konventionellen anatomischen Bildgebungsmethoden eingeschätzt werden [60].

\section{Schilddrüsenkarzinom}

Schilddrüsenkarzinome sind insbesondere bei Kindern unter 15 Jahren mit einer Inzidenz von 1,5\% selten. Die in den meisten Fällen vorliegenden differenzierten Schilddrüsenkarzinome präsentieren sich zum Zeitpunkt der Erstdiagnose deutlich öfter mit Lymphknoten oder Lungenmetastasen als bei Erwachsenen. Trotz in 20-30\% vorliegenden Lungenmetastasen (Erwachsene ca. $2 \%$ ) haben junge Patienten mit Schilddrüsenkarzinom eine exzellente Prognose, was vor allem durch die gute Behandelbarkeit mit ${ }^{131}$ I bedingt ist [63]. Bei ${ }^{131}$ I negativen Metastasen oder Rezidiv kann die PET durch Darstellung der Stoffwechselaktivität (FDG) ein Restaging, bspw. bei ansteigendem Tumormarker, ermöglichen. Komplementär kann bei iod- und FDG-negativen Metastasen die Darstellung der SomatostatinRezeptordichte (DOTATATE/-TOC/-NOC) angewandt werden. Dieses Vorgehen zeigt bei Erwachsenen vielversprechende Ergebnisse, ist jedoch für Kinder, aufgrund der Seltenheit iodnegativer Schilddrüsentumore, nicht abschließend evaluiert [63].

\section{Neurofibromatose Typ I}

Die Neurofibromatose Typ I ist eine autosomal dominant vererbte Multiorganerkrankung, die vor allem die Haut (Café-aulait-Flecken) und das Nervensystem (Neurofibrome) befällt. Eine maligne Transformation, insbesondere plexiformer und 
nodulärer Neurofibrome in einen peripheren Nervenscheidentumor (Weichteilsarkom), ist bei Neurofibromatose Typ I mit einem Risiko von 8-13\% verknüpft [64]. Patienten mit malignen Nervenscheidentumoren und Neurofibromatose Typ I sind dabei deutlich jünger, häufig im Jugendlichen- und jungen Erwachsenenalter, als Patienten mit einem sporadischen malignen Nervenscheidentumor. Aufgrund der schlechten Prognose und der chirurgischen Exzision als Therapie der Wahl im operablen Stadium ist eine frühe Sicherung der Diagnose notwendig $[64,65]$. Die FDG-PET erlaubt durch Darstellung und Quantifizierung des Glukosemetabolismus in vielen Fällen eine Unterscheidung zwischen Neurofibromen und peripheren Nervenscheidentumoren auch im Kindesalter $[66,67]$. Die Möglichkeit der Reduktion der Strahlenexposition bei wiederholten Untersuchungen von Neurofibromatose Typ I Patienten und die Anwendung funktioneller MRT zur zusätzlichen Charakterisierung der verdächtigen Neurofibrome stellen die Vorteile der PET/ MRT bei dieser Erkrankung dar [68].

\section{Pitfalls/Besonderheiten der PET-Bildgebung bei Kindern}

Die Untersuchung von Kindern, insbesondere jüngeren Alters, am PET/CT oder PET/MRT stellt an das medizinische Personal besondere Anforderungen. Kind und Eltern müssen in die Aufklärung und während der Untersuchung miteinbezogen werden. Bei sehr jungen Kindern ist häufig eine Untersuchung in Sedierung oder Kurznarkose erforderlich. In 15-20\% der FDGPET-Untersuchungen kommt es bei Kindern und jungen Erwachsenen zu einer FDG-Aufnahme in das braune Fettgewebe, wodurch Läsionen übersehen oder vorgetäuscht werden können [69]. Bei Aufnahmen mit Hybridsystemen können durch sorgfältige Betrachtung der morphologischen Bildgebung Fehldiagnosen vermieden werden. Um eine Aufnahme in das braune Fettgewebe zu verhindern, wird die Gabe eines Betablockers nach Ausschluss von Kontraindikationen 60 Minuten vor Applikation des Radiopharmakons empfohlen. Weitere Fehldiagnosen können durch Anreicherungen im Thymus entstehen. Bei Kindern und Jugendlichen können Thymusanteile nicht nur mediastinal, sondern auch abgetrennt davon zervikal vorliegen und nur in Kombination mit morphologischen Bildgebungsmethoden sicher diagnostiziert werden.

Die Dosis des injizierten Radiopharmakons hängt von der Sensitivität der PET-Kamera sowie den Akquisitionsparametern ab. Zudem orientiert diese sich am Gewicht des jungen Patienten. Weiterführende Informationen sind den aktuellen Leitlinien der DGN und EANM sowie die „EANM Dosage card“ zu entnehmen.

Bei vielen Tumorerkrankungen des Kindesalters erfolgt eine bioptische Sicherung, häufig schon vor Durchführung des Ganzkörper-Stagings. Operative Zugangswege sowie Manipulationen am Tumor zeigen sich stoffwechselaktiv (FDG-positiv) und können zu einer Überschätzung der Aktivität des Tumors führen. Nach Biopsie können zudem die lokoregionären Lymphknoten reaktiv leicht stoffwechselaktiv zur Darstellung kommen. Teilresektionen des Tumors im Rahmen der Probenent- nahme können zu einer Unterschätzung der Tumoraktivität führen.

Nach Resektion des Primärtumors kann es zu einer erheblichen mechanischen Alteration sowie Veränderung der Statik kommen, die zu langanhaltenden Veränderungen des Stoffwechsels führt, bspw. nach Hemipelvektomie bei einem Ewing-Sarkom im Bereich des Hüftknochens. Eingebrachtes Fremdmaterial kann durch eine Fremdkörperreaktion oder als Folge einer Entzündung einen deutlich erhöhten Stoffwechsel aufweisen.

Weitere Fehlerquellen können die physiologisch erhöhte Stoffwechselaktivität in den Epiphysenfugen sowie im blutbildenden Knochenmark nach Chemotherapie oder Gabe von Granulozyten-koloniestimulierenden Faktoren (G-CSF) sein.

Im Falle einer FDG-PET des Kopfes ist zusätzlich das altersabhängige Anreicherungsmuster des Tracers im Gehirn zu beachten $[69,70]$.

\section{PET/CT-Protokoll}

In der Diagnostik wird bei erwachsenen Patienten häufig eine Rumpfuntersuchung von der Schädelbasis bis zur Mitte des Oberschenkels durchgeführt. Je nach Indikation müssen weitere Regionen mituntersucht werden. Ausnahme bildet die PETDiagnostik des Gehirns bei der lediglich die Akquisition einer Bettposition notwendig ist.

Bei jungen Patienten mit einem Neuroblastom, Sarkom oder Lymphom ist zusätzlich zur Darstellung des Rumpfes eine Abbildung der Arme und Beine notwendig. Grund dafür ist, dass die Knochenmetastasierung über den Markraum stattfindet und das blutbildende Knochenmark bei Kindern deutlich weiter nach distal reicht. Bei FDG-PET-Untersuchungen wird auf die Abbildung des Gehirns wegen der geringen Sensitivität für Hirnmetastasen häufig verzichtet, nicht zuletzt zur Minimierung der Linsendosis in der Niedrigdosis-CT. Kalottenherde können dadurch aber dem Nachweis entgehen. Zumindest bei den Hodgkin-Lymphomen ist ein Verzicht auf die Akquisition des Kopfes aber gerechtfertigt, wobei die Schädelbasis großzügig miterfasst werden muss, um hochzervikale Lymphknoten nicht zu übersehen.

Insgesamt gilt, dass es bei Kindern noch wichtiger ist, die applizierte Aktivität durch hochsensitive PET-Scanner gering zu halten und die Akquisitionszeit zu verkürzen.

Durch die zunehmende Verfügbarkeit von PET-/CT-Systemen und den Eingang der PET-Diagnostik in Leitlinien bspw. bei Staging und Therapiemonitoring des Hodgkin-Lymphoms ist eine genauere Beschäftigung mit den möglichen Risiken einer wiederholten Strahlenexposition notwendig. Bei onkologisch erkrankten Kindern liegt die durchschnittliche kumulative effektive Dosis durch PET/CT-Untersuchungen bei ungefähr $80 \mathrm{mSv}$, für ein einzelnes PET/CT durchschnittlich bei ungefähr 25 mSV [71, 72]. Das führte in der Studie von Nievelstein zu einer Wahrscheinlichkeit für strahlenbedingte Todesfälle durch radiologische und nuklearmedizinische Bildgebung bei Kindern mit Hodgkin-Lymphom von 0,4\% für Jungen und 0,7 \% für Mädchen, im Vergleich zu Erwachsenen mit Non-Hodgkin-Lymphomen von 0,07\% (männlich) und 0,09\% (weiblich) [72]. Der An- 
teil der CT-Komponente an der Strahlenbelastung überwiegt dabei, wobei deutliche Unterschiede je nach Protokoll vorliegen. Eine diagnostische CT-Untersuchung bei Kindern implizierte in der Studie von Chawa et al. im Durchschnitt ungefähr 20 mSV effektive Dosis [71]. Neuere CT-Protokolle mit dem Ziel der Strahlenreduktion kommen auf 5-13 mSv [72] oder sogar 2-10 mSv für ein diagnostisches CT im Rahmen der PET/CT [73]. Die Strahlenbelastung durch ein Ganzkörper-Niedrigdosis-CT liegt demgegenüber unter $3 \mathrm{mSv}$ und die der FDG-PET bei ungefähr 3 bis 8 mSv [71 - 73]. Für CT-Untersuchungen gilt, dass die Wahrscheinlichkeit an einem strahleninduzierten Tumor zu erkranken zwischen den frühen 90er-Jahren und 2000 2008 aufgrund der Strahlenreduktion um 50-70\% auf 0,3-1/ 1000 Patienten für Kopfaufnahmen, sowie 1-5/1000 Patienten für CT des Körperstammes sank [74]. Junges Alter und weibliches Geschlecht erhöhen dabei die Wahrscheinlichkeit, für das weibliche Geschlecht bspw. um das 1,5 bis 3-Fache bei CTs des Körperstammes (Thorax, Abdomen, Pelvis) [74,75]. Das ist verbunden mit einem höheren Risiko für Schilddrüsen-, Lungen-, gynäkologische- und insbesondere Mammakarzinome, da das in der Entwicklung befindliche Brustdrüsengewebe besonders strahlensensibel ist $[72,74,76]$.

Eine Optimierung der Untersuchungsprotokolle, insbesondere der variablen CT-Komponente, sowie die Überprüfung der Notwendigkeit der regelmäßigen Exposition sind somit zwingend erforderlich und führen zu einer größtmöglichen Reduzierung der Strahlenexposition.

Die Anpassung der Röhrenspannung und der Dosisleistung des CT an den kleineren Körperquerschnitt ist unerlässlich. Dosis-Modulationssysteme sollen standardmäßig verwendet werden, um die Strahlenexposition zu minimieren. Bei jedem Patienten muss individuell und je nach vorliegenden Voraufnahmen entschieden werden, ob ein diagnostisches $C T$ einzelner Körperregionen notwendig oder ein Niedrigdosis-CT zur Schwächungskorrektur ausreichend ist. Beim Hodgkin-Lymphom werden häufig diagnostische CTs mit KM von Hals, Abdomen/Becken sowie des Thorax in tiefer Inspiration notwendig. Die Durchführung der Thorax-CT zum Ausschluss von Lungenmetastasen, bspw. bei einem Ewing-Sarkom, sollte als Niedrigdosis-CT in tiefer Inspiration durchgeführt werden [77]. Die Rekonstruktion mit einem Lungenkernel des Niedrigdosis-CT, das zur Schwächungskorrektur angefertigt wird, reicht nicht aus, da in Atemmittellage Rundherde übersehen werden können. Der Einsatz von Röntgenkontrastmittel oder eines diagnostischen CT muss sorgfältig abgewogen werden.

In vielen Fällen ist die deutlich höhere Dosis eines diagnostischen CTs bei nur geringem Zuwachs an Information nicht gerechtfertigt. Sämtliche Hirnuntersuchungen werden inklusive Niedrigdosis-CT zur Schwächungskorrektur oder aber am PET/ MRT durchgeführt.

Neben der Möglichkeit der Anpassung der CT-Komponente der PET/CT-Untersuchung ist die Durchführung eines PET/MRT unter dem Gesichtspunkt der Reduktion der Strahlenexposition möglich.

\section{PET/MRT}

Die Kombination aus klinisch etablierter morphologischer Bildgebung mit hohem Weichteilkontrast und molekularer Ganzkörperdiagnostik ist nicht nur aus strahlenhygienischen Gründen verlockend.

Ziel ist die Evaluation komplementärer Eigenschaften der multiparametrischen MRT mit bspw. Darstellung der Diffusion, Traktografie, T1- oder T2-Mapping, die über die rein morphologische Bildgebung mittels CT hinausgehen. Auf der anderen Seite lassen sich diese Informationen mit quantitativen PETMessungen, wie z.B. Bestimmung von SUV-Werten, MV und TLG vergleichen [6]. Bei ZNS Tumoren bspw. enthalten PET und MRT komplementäre Informationen zu Blut-Hirnschrankenstörungen, funktionellen MR-Parametern, wie Diffusionseinschränkungen und Stoffwechselalterationen in der PET [79, 80]. Eine gleichzeitige Akquisition vereinfacht die Koregistrierung und Bildfusion und senkt die Akquisitionszeit.

Die hochauflösende Lokaldiagnostik durch die Magnetresonanztomografie ist bei Sarkomen ( $\mathbf{A b b} \mathbf{A} \mathbf{1})$, ZNS-Tumoren ( $\triangleright$ Abb.3) und Neurofibromatose Typ I auch bei zusätzlich durchgeführtem PET/CT (Ganzkkörperdiagnostik) unter anderem für die Planung einer Biopsie oder Resektion zwingend erforderlich. Aus diesem Grund erscheint die Kombination beider Verfahren in einer Untersuchung vielversprechend.

Bei Sarkomen im Kindesalter ist trotz steigender Sensitivität für Lungenmetastasen in der MRT die Durchführung eines Niedrigdosis-CT in Inspiration aufgrund der prognostischen Relevanz für das Outcome der Patienten empfohlen [16]. Ein CTThorax wäre zudem für die OP-Planung vor einer geplanten kurativen Resektion von Lungenmetastasen notwendig [80].

Insgesamt gilt, dass die PET-MR-Bildgebung eine äquivalente PET-Diagnostik bei potenziell zusätzlichen Informationen durch die MRT, besonders bei geringer oder heterogener Nuklidanreicherung, liefert. Eine genauere Evaluation der PET/ MRT in großen Studien im Vergleich zum PET/CT ist geboten.

Anmerkung: Diese Arbeit wurde unterstützt von der Deutschen Krebshilfe, Fördernummer 108128 (UD); ERA-NetTRANSCAN Konsortium, Projektnummer 01KT1310 und von David Dressler (UD).

Wolfgang Roll hat eine Rotationsstelle für Forschung der medizinischen Fakultät der Westfälischen Wilhelms-Universität Münster inne.

\section{Literatur}

[1] PDQ Pediatric Treatment Editorial Board PPTE. Childhood NonHodgkin Lymphoma Treatment $\left(\mathrm{PDQ}^{\circledR}\right)$ : Health Professional Version. PDQ Cancer Inf Summ. National Cancer Institute (US); 2002: 1 - 7

[2] PDQ Pediatric Treatment Editorial Board PPTE. Childhood Hodgkin Lymphoma Treatment $\left(\mathrm{PDQ}^{\circledR}\right)$ : Health Professional Version [Internet]. PDQ Cancer Inf. Summ. National Cancer Institute (US); 2002: Available from: http://www.ncbi.nlm.nih.gov/pubmed/26389170

[3] Furth C, Steffen IG, Amthauer H et al. Early and Late Therapy Response Assessment With [ ${ }^{18}$ F]Fluorodeoxyglucose Positron Emission To- 
mography in Pediatric Hodgkin's Lymphoma: Analysis of a Prospective Multicenter Trial. J Clin Oncol 2009; 27: 4385-4391

[4] Flerlage JE, Kelly KM, Beishuizen A et al. Staging Evaluation and Response Criteria Harmonization (SEARCH) for Childhood, Adolescent and Young Adult Hodgkin Lymphoma (CAYAHL): Methodology statement. Pediatr. Blood Cancer 2017; 64: e26421

[5] Cheson BD, Fisher RI, Barrington SF et al. Recommendations for initial evaluation, staging, and response assessment of hodgkin and nonhodgkin lymphoma: The lugano classification. J. Clin. Oncol. American Society of Clinical Oncology 2014; 32: 3059-3067

[6] Tsukamoto N, Kojima M, Hasegawa M et al. The usefulness of (18)Ffluorodeoxyglucose positron emission tomography ((18)F-FDG-PET) and a comparison of (18)F-FDG-pet with (67)gallium scintigraphy in the evaluation of lymphoma: relation to histologic subtypes based on the World Health Organization classification. Cancer 2007; 110 : $652-659$

[7] Cheson BD. PET/CT in Lymphoma: Current Overview and Future Directions. Semin. Nucl. Med. Elsevier 2018; 48: 76 - 81

[8] Meignan M, Gallamini A, Meignan M et al. Report on the First International Workshop on interim-PET scan in lymphoma. Leuk Lymphoma 2009; 50: 1257 - 1260

[9] PDQ Pediatric Treatment Editorial Board PPTE. Childhood Rhabdomyosarcoma Treatment $\left(\mathrm{PDQ}^{\circledR}\right)$ : Health Professional Version. PDQ Cancer Inf. Summ. National Cancer Institute (US); 2002

[10] PDQ Pediatric Treatment Editorial Board PPTE. Osteosarcoma and Malignant Fibrous Histiocytoma of Bone Treatment $\left(P D Q{ }^{\circledR}\right)$ : Health Professional Version. PDQ Cancer Inf. Summ. National Cancer Institute (US); 2002

[11] PDQ PediatricTreatment Editorial Board PPTE. Ewing Sarcoma Treatment $\left(\mathrm{PDQ}^{\circledR}\right)$ : Health Professional Version. PDQ Cancer Inf. Summ. National Cancer Institute (US); 2002

[12] Pappo AS, Dirksen U. Rhabdomyosarcoma, Ewing Sarcoma, and Other Round Cell Sarcomas. J Clin Oncol 2018; 36: 168-179

[13] Franzius C, Daldrup-Link HE, Sciuk J et al. FDG-PET for detection of pulmonary metastases from malignant primary bone tumors: comparison with spiral CT. Ann Oncol Off J Eur Soc Med Oncol 2001; 12 : 479-486

[14] Franzius C, Sciuk J, Daldrup-Link HE et al. FDG-PET for detection of osseous metastases from malignant primary bone tumours: comparison with bone scintigraphy. Eur J Nucl Med 2000; 27: 1305 - 1311

[15] Völker T, Denecke T, Steffen I et al. Positron emission tomography for staging of pediatric sarcoma patients: results of a prospective multicenter trial. J Clin Oncol. American Society of Clinical Oncology 2007; 25: $5435-5441$

[16] Vrachimis A, Schäfers M, Dirksen U et al. Positronenemissionstomografische Diagnostik kindlicher Sarkome. Der Nukl 2014; 37 : 242 249

[17] Hawkins DS, Schuetze SM, Butrynski JE et al. [18F]Fluorodeoxyglucose positron emission tomography predicts outcome for Ewing sarcoma family of tumors. J Clin Oncol 2005; 23: 8828-8834

[18] Denecke T, Hundsdörfer P, Misch D et al. Assessment of histological response of paediatric bone sarcomas using FDG PET in comparison to morphological volume measurement and standardized MRI parameters. Eur J Nucl Med Mol Imaging 2010; 37: 1842 - 1853

[19] Andersen KF, Fuglo HM, Rasmussen SH et al. Semi-Quantitative Calculations of Primary Tumor Metabolic Activity Using F-18 FDG PET/CT as a Predictor of Survival in 92 Patients With High-Grade Bone or Soft Tissue Sarcoma. Medicine (Baltimore) 2015; 94 : e1142

[20] Colleran GC, Kwatra N, Oberg L et al. How we read pediatric PET/CT: indications and strategies for image acquisition, interpretation and reporting. Cancer Imaging 2017; 17: 28
[21] Raciborska A, Bilska K, Drabko K et al. Response to chemotherapy estimates by FDG PET is an important prognostic factor in patients with Ewing sarcoma. Clin Transl Oncol 2016; 18: 189-195

[22] Hawkins DS, Conrad EU, Butrynski JE et al. [F-18]-fluorodeoxy-D-glucose-positron emission tomography response is associated with outcome for extremity osteosarcoma in children and young adults. Cancer 2009; 115: 3519-3525

[23] Gaspar N, Hawkins DS, Dirksen U et al. Ewing Sarcoma: Current Management and Future Approaches Through Collaboration. J Clin Oncol 2015; 33: 3036 - 3046

[24] Haeusler ], Ranft A, Boelling T et al. The value of local treatment in patients with primary, disseminated, multifocal Ewing sarcoma (PDMES). Cancer 2010; 116: 443-50

[25] Newman EN, Jones RL, Hawkins DS. An Evaluation of [F-18]-Fluorodeoxy- D -Glucose Positron Emission Tomography, Bone Scan , and Bone Marrow Aspiration / Biopsy as Staging Investigations in Ewing Sarcoma. Pediatr Blood Cancer 2013; 60: 1113-1117

[26] Treglia G, Salsano M, Stefanelli A et al. Diagnostic accuracy of ${ }^{18} \mathrm{~F}$ FDG-PET and PET/CT in patients with Ewing sarcoma family tumours: a systematic review and a meta-analysis. Skeletal Radiol 2012; 41: $249-256$

[27] Jamet B, Carlier T, Campion L et al. Initial FDG-PET/CT predicts survival in adults Ewing sarcoma family of tumors. Oncotarget. Impact Journals 2017; 8: 77050 - 77060

[28] Palmerini E, Colangeli M, Nanni C et al. The role of FDG PET/CT in patients treated with neoadjuvant chemotherapy for localized bone sarcomas. Eur J Nucl Med Mol Imaging 2017; 44: 215-223

[29] Gupta K, Pawaskar A, Basu S et al. Potential Role of FDG PET Imaging in Predicting Metastatic Potential and Assessment of Therapeutic Response to Neoadjuvant Chemotherapy in Ewing Sarcoma Family of Tumors. Clin Nucl Med 2011; 36: $973-977$

[30] ClinicalTrials.gov. Study in Localized and Disseminated Ewing Sarcoma (EWING2008). 01.10 2009: Available at: https://clinicaltrials. gov/ct2/show/NCT00987636 [Accessed 27 Apr. 2018)

[31] Heinemann M, Ranft A, Langer T et al. Recurrence of Ewing sarcoma: Is detection by imaging follow-up protocol associated with survival advantage? Pediatr Blood Cancer [Internet] 2018: e27011

[32] Heinemann M, Ranft A, Jürgens $\mathrm{H}$ et al. Ewing sarcoma during followup. Nuklearmedizin 2017; 56: $233-238$

[33] Sharma P, Khangembam BC, Suman KCS et al. Diagnostic accuracy of 18F-FDG PET/CT for detecting recurrence in patients with primary skeletal Ewing sarcoma. Eur ] Nucl Med Mol Imaging 2013; 40: 1036 1043

[34] Harrison DJ, Parisi MT, Shulkin BL. The Role of 18 F-FDG-PET/CT in Pediatric Sarcoma. Semin Nucl Med 2017; 47: 229 - 241

[35] Norman G, Fayter D, Lewis-Light $\mathrm{K}$ et al. An emerging evidence base for PET-CT in the management of childhood rhabdomyosarcoma: systematic review. BMJ Open 2015; 5: e006030

[36] Federico SM, Spunt SL, Krasin MJ et al. Comparison of PET-CT and conventional imaging in staging pediatric rhabdomyosarcoma. Pediatr Blood Cancer 2013; 60: 1128-1134

[37] Baum SH, Fruhwald M, Rahbar K et al. Contribution of PET/CT to Prediction of Outcome in Children and Young Adults with Rhabdomyosarcoma. J Nucl Med 2011; 52: 1535 - 1540

[38] Harrison DJ, Parisi MT, Shulkin BL et al. 18F 2Fluoro-2deoxy-D-glucose positron emission tomography (FDG-PET) response to predict eventfree survival (EFS) in intermediate risk (IR) or high risk (HR) rhabdomyosarcoma (RMS): A report from the Soft Tissue Sarcoma Committee of the Children's Oncolog. American Society of Clinical Oncology; 2016

[39] Casey DL, Wexler LH, Fox J] et al. Predicting Outcome in Patients with Rhabdomyosarcoma: Role of [18F]Fluorodeoxyglucose Positron Emission Tomography. Int J Radiat Oncol 2014; 90: 1136-42 
[40] PDQ Pediatric Treatment Editorial Board PPTE. Neuroblastoma Treatment $\left(\mathrm{PDQ}^{\circledR}\right)$ : Health Professional Version. PDQ Cancer Inf. Summ. National Cancer Institute (US); 2002

[41] Melzer HI, Coppenrath E, Schmid I et al. 123I-MIBG scintigraphy/ SPECT versus $18 \mathrm{~F}$-FDG PET in paediatric neuroblastoma. Eur ] Nucl Med Mol Imaging 2011; 38: 1648-1658

[42] Gatidis S, Bender B, Reimold M et al. PET/MRI in children. Eur. J. Radiol 2017; 42: A64 - A70

[43] Alexander N, Vali R, Ahmadzadehfar $\mathrm{H}$ et al. Review: The role of radiolabeled DOTA-conjugated peptides for imaging and treatment of childhood neuroblastoma. Curr Radiopharm 2017; 11: 14-21

[44] Ostrom QT, Gittleman H, Fulop J et al. CBTRUS Statistical Report: Primary Brain and Central Nervous System Tumors Diagnosed in the United States in 2008-2012.

[45] Plotkin M, Guggemos A, Grosse F et al. Rolle der PET in der Diagnostik von Hirntumoren im Kindes- und Jugendalter. Der Nukl 2014; 37: $254-257$

[46] Herholz K, Pietrzyk U, Voges ] et al. Correlation of glucose consumption and tumor cell density in astrocytomas. A stereotactic PET study. J Neurosurg 1993; 79: $853-858$

[47] Plotkin M, Blechschmidt C, Auf G et al. Comparison of F-18 FET-PET with F-18 FDG-PET for biopsy planning of non-contrast-enhancing gliomas. Eur Radiol 2010; 20: 2496-2502

[48] Messing-Jünger AM, Floeth FW, Pauleit D et al. Multimodal target point assessment for stereotactic biopsy in children with diffuse bithalamic astrocytomas. Child's Nerv Syst 2002; 18: 445 - 449

[49] Piroth MD, Galldiks N, Pinkawa M et al. Relapse patterns after radiochemotherapy of glioblastoma with FET PET-guided boost irradiation and simulation to optimize radiation target volume. Radiat Oncol 2016; 11: 87

[50] Poulsen SH, Urup T, Grunnet K et al. The prognostic value of FET PET at radiotherapy planning in newly diagnosed glioblastoma. Eur J Nucl Med Mol Imaging 2017; 44: 373-381

[51] Di Chiro G, DeLaPaz RL, Brooks RA et al. Glucose utilization of cerebral gliomas measured by [18F] fluorodeoxyglucose and positron emission tomography. Neurology 1982; 32: $1323-1329$

[52] Misch M, Guggemos A, Driever PH et al. 18F-FET-PET guided surgical biopsy and resection in children and adolescence with brain tumors. Child's Nerv Syst 2015; 31: 261-267

[53] Dunkl V, Cleff C, Stoffels G et al. The Usefulness of Dynamic O-(2-18FFluoroethyl)-L-Tyrosine PET in the Clinical Evaluation of Brain Tumors in Children and Adolescents. J Nucl Med 2015; 56: 88 - 92

[54] Weckesser M, Langen KJ, Rickert CH et al. O-(2-[18F]fluorethyl)-L-tyrosine PET in the clinical evaluation of primary brain tumours. Eur J Nucl Med Mol Imaging 2005; 32: $422-429$

[55] Rachinger W, Goetz C, Pöpperl G et al. Positron emission tomography with O-(2-[18F]flouroethyl)-L- tyrosine versus magnetic resonance imaging in the diagnosis of recurrent gliomas. Neurosurgery 2005; 57: $505-511$

[56] Pöpperl G, Götz C, Rachinger W et al. Value of O-(2-[18F]fluoroethyl)L-tyrosine PET for the diagnosis of recurrent glioma. Eur J Nucl Med Mol Imaging 2004; 31: $1464-1470$

[57] Pöpperl G, Goldbrunner R, Gildehaus FJ et al. O-(2-[18F]fluoroethyl)L-tyrosine PET for monitoring the effects of convection-enhanced delivery of paclitaxel in patients with recurrent glioblastoma. Eur J Nucl Med Mol Imaging 2005; 32: $1018-1025$

[58] Plotkin M, Guggemos A, Steffen I et al. Prospektive, multizentrische Studie zur Bedeutung der O-(2-[18F]Fluoroethyl)-L-Tyrosin-Positronen-Emissions-Tomografie (FET-PET) in der Verlaufsbeurteilung von Hirntumoren im Kindes- und Jugendalter (FET PET 2010). Der Nukl. 2011; 34: $125-129$
[59] PDQ Pediatric Treatment Editorial Board PPTE. Langerhans Cell Histiocytosis Treatment $\left(\mathrm{PDQ}^{\circledR}\right)$ : Health Professional Version. PDQ Cancer Inf. Summ. National Cancer Institute (US); 2002

[60] Phillips M, Allen C, Gerson P et al. Comparison of FDG-PET scans to conventional radiography and bone scans in management of Langerhans cell histiocytosis. Pediatr Blood Cancer 2009; 52: 97-101

[61] Kaste SC, Rodriguez-Galindo C, McCarville ME et al. PET-CT in pediatric Langerhans cell histiocytosis. Pediatr Radiol 2007; 37: 615-622

[62] Sher AC, Orth R, McClain K et al. PET/MR in the Assessment of Pediatric Histiocytoses. Clin Nucl Med 2017; 42: $582-588$

[63] Francis GL, Waguespack SG, Bauer A] et al. Management Guidelines for Children with Thyroid Nodules and Differentiated Thyroid Cancer The American Thyroid Association Guidelines Task Force on Pediatric Thyroid Cancer. Thyroid Am Thyroid Assoc 2015; 25: 716 - 759

[64] Evans DGR, Baser ME, McGaughran J et al. Malignant peripheral nerve sheath tumours in neurofibromatosis 1. J Med Genet 2002; 39: 311 314

[65] Ferner RE, Huson SM, Thomas $\mathrm{N}$ et al. Guidelines for the diagnosis and management of individuals with neurofibromatosis 1. J Med Genet. BM] Publishing Group 2007; 44: 81 - 88

[66] Tsai LL, Drubach L, Fahey F et al. [18F]-Fluorodeoxyglucose positron emission tomography in children with neurofibromatosis type 1 and plexiform neurofibromas: Correlation with malignant transformation. J Neurooncol 2012; 108: 469-475

[67] Azizi AA, Slavc I, Theisen BE et al. Monitoring of plexiform neurofibroma in children and adolescents with neurofibromatosis type 1 by $\left[{ }^{18} \mathrm{~F}\right]$ FDG-PET imaging. Is it of value in asymptomatic patients? Pediatr Blood Cancer 2018; 65: e26733

[68] Gatidis S, Gückel B, la Fougère C et al. Simultane Ganzkörper-PET-MRT in der pädiatrischen Onkologie: Mehr als nur Strahlenersparnis? Radiologe 2016; 56: $622-630$

[69] Stauss J, Franzius C, Pfluger T et al. Guidelines for 18F-FDG PET and PET-CT imaging in paediatric oncology. Eur J Nucl Med Mol Imaging. 2008; 35: $1581-1588$

[70] Chugani HT. A Critical Period of Brain Development: Studies of Cerebral Glucose Utilization with PET. Prev Med (Baltim) 1998; 27: 184 188

[71] Chawla SC, Federman N, Zhang D et al. Estimated cumulative radiation dose from PET/CT in children with malignancies: a 5-year retrospective review. Pediatr Radiol. 2010; 40: 681-686

[72] Nievelstein RAJ, Quarles van Ufford HME, Kwee TC et al. Radiation exposure and mortality risk from CT and PET imaging of patients with malignant lymphoma. Eur Radiol 2012; 22: 1946 - 1954

[73] Parisi MT, Bermo MS, Alessio AM et al. Optimization of Pediatric PET/ CT. Semin Nucl Med 2017; 47: 258-274

[74] Journy NMY, Lee C, Harbron RW et al. Projected cancer risks potentially related to past, current, and future practices in paediatric $\mathrm{CT}$ in the United Kingdom, 1990-2020. Br J Cancer. Nature Publishing Group 2017; 116: 109-116

[75] Miglioretti DL, Johnson E, Williams A et al. The Use of Computed Tomography in Pediatrics and the Associated Radiation Exposure and Estimated Cancer Risk. JAMA Pediatr. American Medical Association 2013; 167: 700

[76] De Bruin ML, Sparidans ], van't Veer MB et al. Breast Cancer Risk in Female Survivors of Hodgkin's Lymphoma: Lower Risk After Smaller Radiation Volumes. J Clin Oncol 2009; 27: 4239-4246

[77] Juergens KU, Weckesser M, Stegger L et al. Tumor staging using whole-body high-resolution 16-channel PET-CT: does additional lowdose chest $\mathrm{CT}$ in inspiration improve the detection of solitary pulmonary nodules? Eur Radiol 2006; 16: 1131-1137 
[78] Morana G, Piccardo A, Tortora D et al. Grading and outcome prediction of pediatric diffuse astrocytic tumors with diffusion and arterial spin labeling perfusion MRI in comparison with 18F-DOPA PET. Eur J Nucl Med Mol Imaging 2017; 44: $2084-2093$

[79] Zukotynski KA, Vajapeyam S, Fahey FH et al. Correlation of 18F-FDC PET and MRI Apparent Diffusion Coefficient Histogram Metrics with
Survival in Diffuse Intrinsic Pontine Glioma: A Report from the Pediatric Brain Tumor Consortium. J Nucl Med 2017; 58: 1264 - 1269

[80] Fuchs J, Seitz G, Handgretinger R et al. Surgical treatment of lung metastases in patients with embryonal pediatric solid tumors: an update. Semin Pediatr Surg 2012; 21: 79-87 